\title{
Bandwidth Efficient GNDA
}

\author{
Uma Narayanan ${ }^{1}$, Arun Soman ${ }^{2}$ \\ ${ }^{1}$ (Dept of Information Technology Rajagiri School of Engineering \& Technology, M. G University) \\ ${ }_{2}^{2}$ (Assistant Professor, Dept of Information Technology Rajagiri School of Engineering \& Technology, M.G \\ University)
}

\begin{abstract}
With the tremendous advent of internet and related technologies the significance of adhoc networks are increasing day by day. Adhoc networks are infrastructure less, self-creating wireless networks. Since nodes are mobile and topology changes dynamically route selection becomes very difficult. This type of network is easily vulnerable to internal and external attacks due to the presence of bad neighbor nodes. Identification of nodes as good or bad is based on its flow capacity, signal strength and relative positioning of nodes in the network. Also due to the high mobility of nodes energy consumption is also more. Power is one of the most important design criteria for adhoc networks as batteries provide limited working capability to the mobile nodes. Power failure of a mobile node not only affects the node itself but also its ability to forward packets on behalf of others and hence affects the overall network lifetime. In this paper we are introducing two concepts for efficient selection of good neighbor nodes and effective bandwidth selection for routing.
\end{abstract}

Keywords: AODV, MANET, Bandwidth Efficient GNDA, Traffic

\section{INTRODUCTION}

In this paper, we are proposing that consideration of good neighbor nodes increases the performance of the network. Good neighbor means it can forward the data with the same amount as such it receives it. Also it has sufficient signal strength to forward the packet. So Selection of a node as good or bad neighbor depends on signal strength, flow capacity of nodes and also, how fast each node can receive the complete information. Proposed approach is analyzed by using AODV routing protocol.

This paper also addresses the problem of power awareness routing to increase lifetime of overall network. Since nodes are moving randomly, the network topology is changing frequently. This frequent movement and forwarding of packets results in energy wastage and hence degrade network performance. Therefore it is very difficult to find and maintain an optimal power aware route. In this paper a scheme has been proposed to maximize the network lifetime and minimizes the power consumption during the source to destination route establishment.

We are experimenting the work on AODV protocol. Adhoc on demand distance vector routing protocol is an on demand routing protocol.ie, routing information is not stored in tables and hence no need of table updating and other overheads. In this protocol, routing discovery process is initiated only when route is required. Whenever a node want to send a packet it initializes a Route Request (RREQ) and send to the network. It propagates until it reaches the destination. Destination selects the shortest path and sends a Route Reply (RREP) through the selected shortest path until it reaches the source node. After getting this RREP packet source node sends the data. If source node or intermediate node moves then the moving nodes realizes link failure and sends a Route Error (RERR) notification to its upstream neighbors until it reaches the source. So, source can reinitiate the Route Request if needed.

\section{RELATED WORKS}

Since MANET is infrastructure less security is the main issue. In order to overcome this issue we use good neighbor nodes to send the packet. Since MANET runs on battery which is a scare resource, only considering good neighbor nodes will not solve the problem. So we have to calculate energy efficient path also. Different energy-related metrics that have been used to determine energy efficient routing path: Energy consumed/ packet, Time to network partition, Variance in node power levels, Cost/packet, and Maximum node cost. In AODV, Nodes $i$ and $j$ are said to be neighbors if the received transmission power of one of them and maximum power exceeds some given threshold $\tau$. Local and global connectivity of a network increases when good neighbors are present throughout the network.

\section{PROPOSED SYSTEM: BANDWIDTH EFFICIENT GNDA}

In this approach, initially all nodes maintain their own transmission range. We are comparing Transmission range (NTr) of each node present in the network with the total transmission of network (TTrN). Determination 
of transmission power is required to send a message between node $\mathrm{n}$ and its neighbor $\mathrm{n} 1$. It can be calculated by measuring the received power of hello message. When node $\mathrm{n}$ receives hello messages from a neighbor node $\mathrm{n} 1$, it can estimate the minimum power level needed to reach $n 1$ by comparing the received power of hello message with maximum transmit power. This method is achieved by considering some additional parameters in the neighbor table such as flow capacity, signal strength and residual power. Address of node is stored into the neighbor table based on their transmission range. If (NTr > TTrN), then adjust energy of this node accordingly. Otherwise calculate signal strength by using equation (1). This is the first step. Next step is calculating the flow capacity of a node as mentioned in equation (2). If flow capacity and signal strength of a node is good then store address of the node otherwise remove the address from routing table.

After this calculation we are identifying the shortest path among the selected good neighbor nodes. After receiving the first RREQ packet the destination wait for a random amount of time to receive multiple RREQ packets. After the time out destination processes the RREQ packet and identifies the path with maximum power and send back the RREP packet to the corresponding source node. Then the source node can transmit the data packets through the energy efficient path which satisfies the maximum delivery rate. If sufficient battery power is not present then send a negative Acknowledgement.

Following are the parameters for deciding whether the node is good or bad.

1. Signal Strength

Signal Strength of a node is computed by the formula which is as follows:

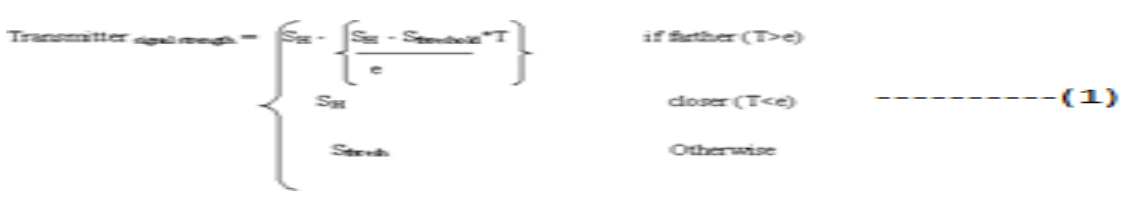

Where SH signal strength of hello message and $\mathrm{T}$ is is the time period between two successive hello packets and $\mathrm{e}$ is the link connectivity between $\mathrm{i}$ and $\mathrm{j}$.

2. Flow Capacity

The capacity of directed edge is denoted as Cij source s and destination $\mathrm{d}$. F is assumed as a flow in $\mathrm{G}$ where $\mathrm{E}$ belongs to edge (i,j). If for all $(i, j) \in E ; 0<=$ Fij $<=$ Cij; s.t.

$$
\sum_{j=(s, j) \in E} F_{s j-} \sum_{i=(i, s) \in E} F_{i=1}
$$

Let Fis and Fsj be the counter of amount of bytes that flowed on the link (i, j) upto time $t$ in packets. Thus, If signal strength range is negligible then discard this node and delete entry of this node from the neighbor table. Otherwise calculate flow capacity of a node by considering equation (2). Based on flow capacity and packet delivery ratio, good neighbors are identified.

\section{Traffic less routing}

The proposed algorithm maximizes the network lifetime \& minimizes the power consumption during the source to destination route establishment.

\section{a) Parameters to Concern during Route Search}

At the time of route discovery, a route request (RREQ) packet broadcasted by the source. The header of the RREQ packet includes source_id, destination_id, $T \_O \_D$ (type of data to be transfer), T_ $T \_L$ (Total Traffic Level), and Node_IDs.

b) Calculation of Total Traffic level $\left(T_{-} T_{-} L\right)$ :

1) At a source node, Initially $T_{-} T \_L=0$.

2) At the time of route discovery, add traffic status of each intermediate node to T_T_L.

\section{c) Route Selection Criteria at Destination Side}

The destination waits for a threshold time (Tth) after a RREQ packet arrives. During that time, the destination determines T_T_L of the route for every arrived RREQ packet. Destination stores all possible route request for a certain amount of time. When the complete timer expires the destination node selects the route with the minimum $T_{-} T_{-} L$ and replies for a path accordingly. 


\section{Algorithm}

STEP 1: Initialize Total number of nodes in the network

STEP 2: Initialize TTr of the network

STEP 3: Broadcast Hello message

STEP 4: Receive Hello message

STEP 5: Calculate time, of reaching Hello message

STEP 6: Compare NTr and TTr

STEP 6.1: if NTr > TTr then Decrease the NTr and go to step: 6

STEP 6.2: else go to step 7

STEP 7: Calculate signal strength

STEP 7.1: I f signal strength > = Threshold then go to step: 8

STEP 7.2: else it is a weak signal so go to step: 4

STEP 8: Calculate flow capacity

STEP 8.1: If flow capacity is ok then store node address (Good node)

STEP 8.2: else Bad node

STEP 9: Send RREQ through good node

STEP 10: When RREQ reach the Destination, it stores the T_T_L of route.

STEP 10.1: After the time out the destination identifies the RREQ with minimum Total Traffic Level then Select the path one with Minimum T_T_L for RREP and acknowledge the source with the selected path.

STEP 10.2: else if all route have high $T_{-} T_{-} L$ greater than threshold then Send negative acknowledgement to the source that path cannot be established now, Wait random amount time and then send RREQ.

STEP 11: Send Data if path available

STEP 12: Stop

\section{CONCLUSION}

Energy efficiency is one of the main problems in a mobile ad hoc network, especially designing a routing protocol. The proposed work aims at discovering an efficient energy aware routing scheme in MANETs. Although this scheme can somewhat enhance the latency of the data transfer but it results in a significant power saving and long lasting routes. This scheme is one of its types in adhoc networks which can provide different routes for different type of data transfer and ultimately increases the network lifetime. All information related with good neighbors are stored in routing table which improves performance of routing protocol in terms of good communication and stable route. Analytical results of proposed solution shows that it improves data throughput, improve overall performance of the network and improve network life with in fixed and dynamic transmission range.

Simulation result is shown below in which proposed one show significant improvement when compared with existing. Throughput of proposed, Bandwidth Efficient GNDA is above $95 \%$ when compared with the AODV. The Energy usage of Proposed is also low when compared with AODV.

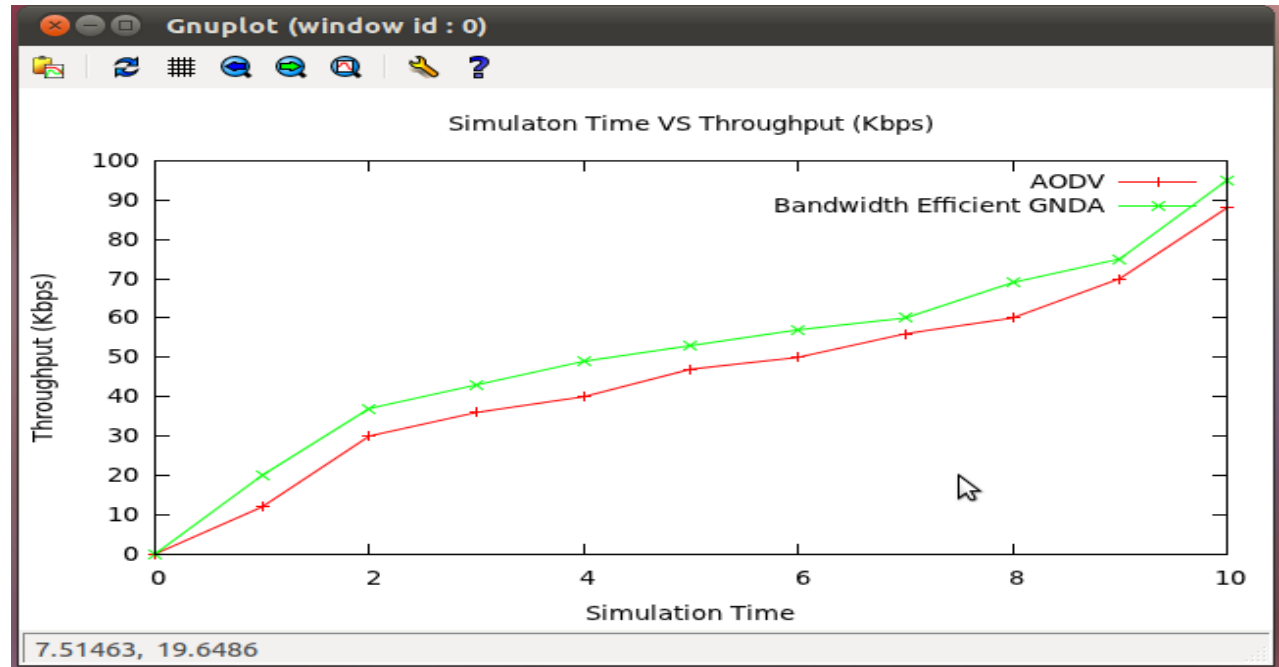

Fig 1:Simulation Result of Throughput between AODV vs Bandwidth Efficient GNDA 


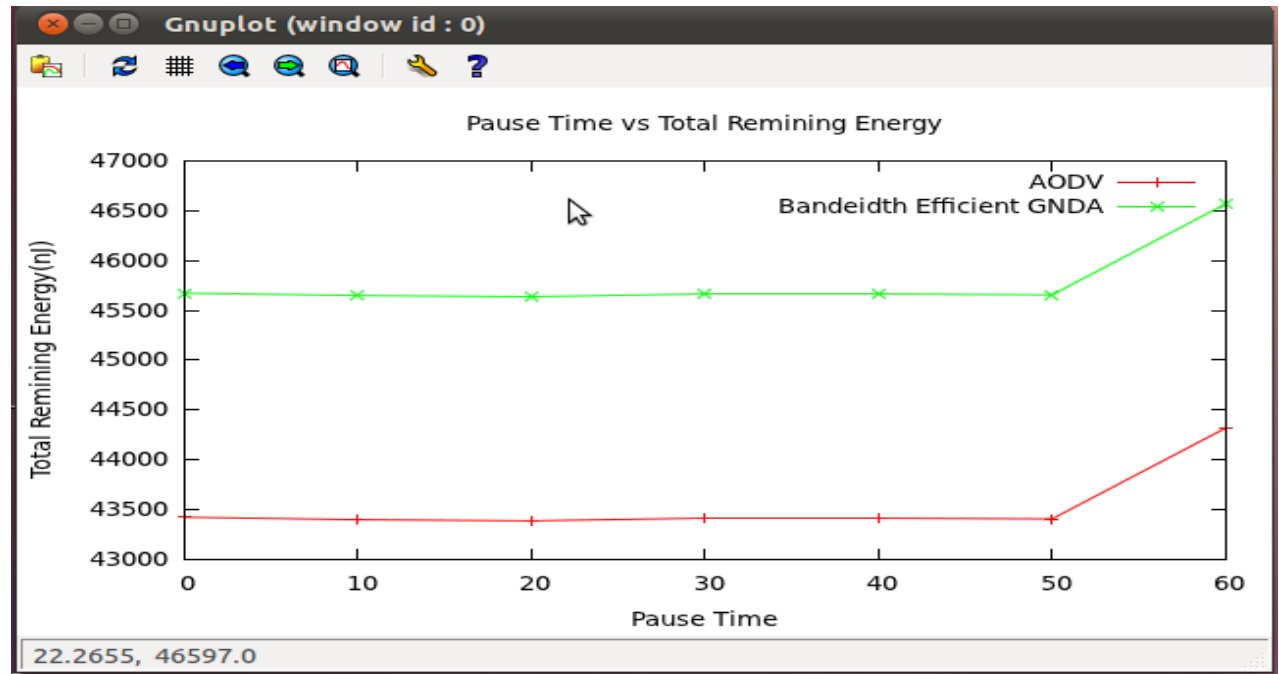

Fig 2: Simulation Result of Remaning Energy level between AODV vs Bandwidth Efficient GNDA

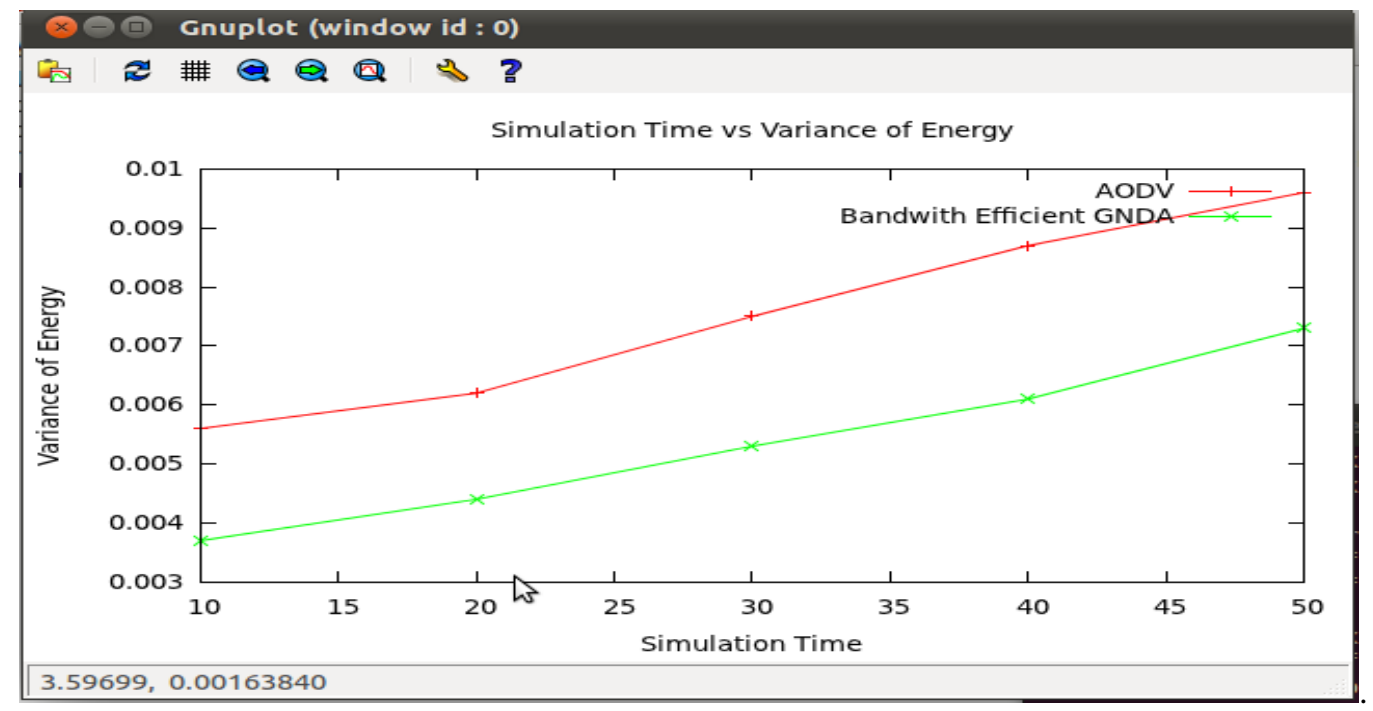

Fig 3: Simulation Result of Energy usage between AODV vs Bandwidth Efficient GNDA

\section{REFERENCES}

[1] Umang Singh, B. V. R. Reddy,M. N. Hoda,"GNDA: Detecting good neighbor nodes in adhoc routing protocol", Second International Conference on Emerging Applications of Information Technology,2011.

[2] H. Yang, X. Meng, and S. Lu, Self-Organized Network-Layer Security in Mobile Ad hoc Networks. International Conference on Mobile Computing and Networking, Atlanta, GA, USA, 2002, 11-20.

[3] Y.-C. Hu, A. Perrig, and D. B. Johnson, Efficient Security Mechanisms for Routing Protocols. Network and Distributed System Security Symposium, NDSS '03, SanDiego, USA, 2003, 57-73.

[4] Chang J-H, Tassiulas L, "Energy Conserving Routing in Wireless Ad-hoc Networks," Proc. IEEE International Conf. On Computer Communications (IEEE Infocom) 2000;

[5] C.Siva Ram Murthy and B.S.Manoj, “Ad hoc Wireless Networks”, Pearson 2005.ISBN 81-297-0945- 7.

[6] P.Santi, "Topological Control in Wireless Adhoc and Sensor Networks", John Wiley \& Sons Ltd., 2005.

[7] Srdjan Krco and Marina Dupcinov, "Improved Neighbor Detection Algorithm for AODV Routing Protocol”,IEEE COMMUNICATIONS LETTERS, VOL. 7, NO.12, DECEMBER2003.

[8] Vinay Rishiwal, Mano Yadav and Shekhar Verma, “ Power Aware Routing to Support Real Time Traffic in Mobile Adhoc Networks ",First International Conference on Emerging Trends in Engineering and Technology, 2009 